DOI: $10.17516 / 1999-494 X-0351$

УДК 629.783

\title{
Relativistic Effect in Simulators \\ of Satellite Radio Navigation Systems
}

\author{
Igor N. Sushkin**, Vladimir A. Kopylova, \\ Roman A. Ruf ${ }^{b}$ and Dmitriy E. Korshunov ${ }^{b}$ \\ ${ }^{a}$ Siberian Federal University \\ Krasnoyarsk, Russian Federation \\ ${ }^{b}$ Military unit 15644 \\ Znamensk, Russian Federation
}

Received 17.05.2021, received in revised form 12.07.2021, accepted 03.09.2021

\begin{abstract}
The paper considers the method of forming the correction factor due to the relativistic effect in the formation of pseudorange in the simulators of satellite radio navigation systems. This correction factor is formed for each navigation spacecraft and can reach units of meters.
\end{abstract}

Keywords: satellite radio navigation systems, GLONASS, GPS, radio navigation signal simulator, relativistic effect, relativistic effect.

Citation: Sushkin I. N., Kopylov V.A., Ruf R. A., Korshunov D. E. Relativistic Effect in Simulators of Satellite Radio Navigation Systems, J. Sib. Fed. Univ. Eng. \& Technol., 2021, 14(7), 854-859. DOI: 10.17516/1999-494X-0351

(C) Siberian Federal University. All rights reserved

This work is licensed under a Creative Commons Attribution-Non Commercial 4.0 International License (CC BY-NC 4.0).

* Corresponding author E-mail address: isushkin@sfu-kras.ru 


\title{
Учет релятивистского эффекта
}

\section{в имитаторах спутниковых \\ радионавигационных систем}

\author{
И.Н. Сушкина \\ Р. А. Руф ${ }^{\tilde{\sigma}}$, Д. Е. Коршунов ${ }^{\tilde{\sigma}}$ \\ ${ }^{a}$ Сибирский федеральный университет \\ Российская Федераиия, Красноярск \\ ${ }^{\sigma} 6 / 415644$ \\ Российская Федерация, Знаменск
}

\begin{abstract}
Аннотация. В статье рассматривается метод формирования поправочного коэффициента, обусловленного релятивистским эффектом при формировании псевдодальности в имитаторах спутниковых радионавигационных систем. Данный поправочный коэффициент формируется для каждого навигационного космического аппарата и может достигать единиц метров.

Ключевые слова: спутниковые радионавигационные системы, ГЛОНАCC, GPS, имитатор радионавигационных сигналов, релятивистский эффект.

Цитирование: Сушкин, И.Н. Учет релятивистского эффекта в имитаторах спутниковых радионавигационных систем / И.Н. Сушкин, В. А. Копылов, Р. А. Руф, Д.Е. Коршунов // Журн. Сиб. федер. ун-та. Техника и технологии, 2021, 14(7). С. 854-859. DOI: 10.17516/1999-494X-0351
\end{abstract}

Постановка проблемы. В настоящее время широкое распространение получила навигационная аппаратура потребителя (НАП), работающая по сигналам спутниковых радионавигационных систем ГЛОНАСС и GPS как гражданского, так и военного применения. В процессе разработки алгоритмов обработки радионавигационных сигналов остро стоит проблема проверки работоспособности и заявленных точностных характеристик. С этой целью, а также при поверке НАП необходимо использование имитатора спутниковых радионавигационных сигналов [1].

Постановка задачи. При формировании псевдодальности в имитационных системах ставится задача учета влияния релятивистского эффекта. Релятивистская составляющая нестабильности частоты генератора, применяемого на борту навигационного космического аппарата, вызвана влиянием изменяющегося гравитационного поля Земли. Нестабильность бортового генератора приводит к рассинхронизации часов навигационного космического аппарата с системным синхронизатором. Данная величина индивидуальна для каждого НКА и может достигать единиц наносекунд.

Формирование псевдодальности имитатором навигационных сигналов (НC) от $i$-го навигационного космического аппарата (НКА) до навигационной аппаратуры потребителя производится по формуле [2]

$$
\tilde{R}_{i}=R_{i}+c \cdot \tau_{i, \text { oon }}+c \cdot \tau_{i, t r}+c \cdot \Delta T_{i, s}+c \cdot \tau_{i, r e c}+R_{i, e f},
$$

где $R_{i}$ - геометрическая дальность от $i$-го НКА до НАП, определяемая по формуле $R_{i}=\sqrt{\left(x_{c, i}-x\right)^{2}+\left(y_{c, i}-y\right)^{2}+\left(z_{c, i}-z\right)^{2}}, x_{\mathrm{c}, i}, y_{\mathrm{c}, i}, z_{\mathrm{c}, i}-$ координаты $i$-го НКА, $x, y, z$ - координаты НАП; $c$ - скорость света; $\tau_{i, \text { ion }}$ - задержка распространения сигнала в ионосферном слое Земли

$$
-855-
$$


от $i$-го НКА до НАП; $\tau_{i, t r}$ - задержка распространения сигнала в тропосферном слое Земли от $i$ го НКА до НАП; $V T_{i, s}$ - отклонение часов $i$-го НКА от часов Центрального синхронизатора; $\tau_{i, \text { rec }}$ - задержка распространения сигнала приемного тракта НАП; $R_{i, \text { ef }}$ - корректировка псевдодальности, вызванная гравитационным и релятивистским эффектом.

Для учета поправочного коэффициента псевдодальности, обусловленного релятивистским эффектом, необходимо произвести коррекцию часов навигационного космического аппарата.

В соответствии с интерфейсным контрольным документом ГЛОНАСС [3] для описания ухода шкалы времени бортовых часов НКА $\Delta T_{S}(t)$ относительно времени Центрального синхронизатора $T_{\text {ЦС }}(t)$ принята математическая модель нестабильности в виде дифференциального уравнения

$$
\Delta f(t)=s(t)+\omega(t)+F_{r e l}(t)
$$

где $\Delta f(t)$ - относительное отклонение частоты бортового генератора от генератора Центрального синхронизатора; $s(t)$ - долговременная составляющая нестабильности генератора на интервале времени не менее одного часа; $\omega(t)$ - кратковременная составляющая нестабильности генератора; $F_{r e l}(t)$ - нестабильность, вызванная гравитационным и релятивистским эффектом.

В дискретном виде уход шкалы времени $V T\left(t_{k}\right)$ на интервале времени $\mathrm{t} \in\left[t_{k}, t_{k+1}\right]$ представляется равенством

$$
\begin{aligned}
& \Delta T\left(t_{k+1}\right)=\Delta T\left(t_{k}\right)+s(t) \cdot\left(t-t_{k}\right)+\int_{t_{k}}^{t_{k+1}} \omega(\tau) d \tau+\int_{t_{k}}^{t_{k+1}} F_{r e l}(\tau) d \tau . \\
& \Delta \dot{T}(t)=\lim _{h \rightarrow 0} \frac{\Delta T\left(t_{k+1}\right)-\Delta T\left(t_{k}\right)}{t_{k+1}-t_{k}}, h=t_{k+1}-t_{k} .
\end{aligned}
$$

Долговременная составляющая нестабильности бортового генератора $s(t)$ представляется гладкой функцией, допускающей параметрическое разложение в той или иной системе базисных функций [4]. Как правило, долговременную составляющую нестабильности частоты хорошо описывают модели полиномиального типа. Кратковременная составляющая нестабильности бортового генератора $\omega(t)$ представляется в виде выходного сигнала фильтра, на входе которого действует «белый шум» с ограниченной дисперсией.

Для уравнения (1) долговременная составляющая нестабильности $s(t)$ представляется на интервале времени $\left[t_{k}, t_{k+1}\right]$ линейной комбинацией

$$
s(t)=\sum_{i=0}^{n} \phi_{i}(t) \cdot a_{i}+F_{r e l}(t)
$$

где $\phi_{i}(t)$ - базисные функции; $a_{i}$ - неизвестные постоянные на интервале времени $\left[t_{k}, t_{k+1}\right]$, которые подлежат оцениванию для каждого типа генератора. В качестве базисных функций применим ортогональный полином Чебышева, для расчета которых применяются следующие выражения:

$$
\begin{aligned}
& \phi_{0}(t)=1, \\
& \phi_{1}(t)=t-\frac{1}{N} \sum_{i=0}^{N-1} t_{i}
\end{aligned}
$$




$$
\phi_{2}(t)=t^{2}-\frac{1}{N} \sum_{i=0}^{N-1} t_{i}^{2}-\frac{\sum_{i=0}^{N-1} t_{i}^{2} \cdot \phi_{1}\left(t_{i}\right)}{\sum_{i=0}^{N-1} \phi_{1}^{2}\left(t_{i}\right)} \cdot \phi_{1}(t), t_{i} \in\left[t_{k} ; t_{k+1}\right],
$$

где $N$ - число измерений на интервале $\left[t_{k}, t_{k+1}\right]$.

При аппроксимации дискретных значений исследуемого процесса с помощью полиномов Чебышева можно перейти к степенным полиномам. При этом выражение (1) будет иметь вид

$$
s(t)=a_{s 0}+a_{s 1}\left(t_{k+1}-t_{k}\right) \cdot t+a_{s 2} \cdot\left(t_{k+1}-t_{k}\right)^{2}+F_{r e l}(t), \quad t \in\left[t_{k+1}-t_{k}\right] .
$$

При этом коэффициенты разложения $a_{\mathrm{i}}$ пересчитываются в коэффициенты степенных полиномов $a_{s i}$ и имеют следующий смысл:

- $a_{s 0}$ - смещение шкал времени;

- $a_{s 1}$ - относительное отклонение частот;

- $a_{s 2}$ - дрейф частоты на интервале $\left[t_{k}, t_{k+1}\right]$.

Значения коэффициентов $a_{s i}$ передаются навигационным космическим аппаратом в качестве частотно-временных поправок (ЧВП).

При применении имитационных систем для проверки работоспособности, отработке алгоритмов и программного обеспечения, а также поверки метрологических характеристик навигационной аппаратуры потребителя ставится задача учета релятивистских эффектов. Причинами этих эффектов являются гравитационное поле Земли и его неоднородность, гравитационные поля соседних небесных тел, неравномерность вращения Земли, высокие скорости измерителей и др. Уровень относительных релятивистских измерений интервалов времени, расстояний и частоты электромагнитных волн в околоземном пространстве достигает $10^{-9}-10^{-}$ ${ }^{10}$, что на 5-6 порядков превышает достигнутые на сегодняшний день относительные инструментальные погрешности эталонов времени и частоты [5].

Релятивистская составляющая $F_{r e l}(t)$ нестабильности частоты из уравнения (1) вызвана влиянием изменяющегося гравитационного поля Земли. Значения уходов часов

$$
\int_{t_{k}}^{t_{k+1}} F_{r e l}(\tau) d \tau=\Delta T_{c o n}+\Delta T_{\sim}
$$

где $V T_{\text {con }}$ - постоянное смещение часов, зависящее от величины главной полуоси орбиты НКА, и равно около 37 мкс/сут [3]; $V T_{i}$ - периодическое смещение шкалы времени, зависящее от параметров движения НКА (эксцентриситета, угловой аномалии, главной полуоси орбиты).

Релятивистский эффект является причиной замедления хода бортовых часов. Принято разделять для ГНСС две составляющие:

1) Постоянная составлявшая, представляет собой смещение частоты, компенсируется как пропорциональное смещение частоты для всех опорных генераторов ГЛОНАСС $\mathrm{V} f / f=-4,36$. $10^{-10}$ и для GPS $\mathrm{V} f / f=-4,47 \cdot 10^{-10}$ согласно интерфейсным контрольным документам. Эта поправка незначительно изменяется от спутника к спутнику, так как зависит от главной полуоси орбиты, служащей причиной смещения часом наблюдаемого НКА.

2) Периодическая составляющая, которая в основном зависит от эксцентриситета орбиты, в равной степени соответствует общим и специальным релятивистским эффектам (откло- 
нение высоты и скорости НКА от средних значений). Величина специального релятивистского эффекта (обусловленная средней скоростью НКА) обычно включается в постоянную часть.

Общепринятая периодическая погрешность, применяемая всеми потребителями высокоточных GPS, рассчитывается как

$$
\Delta t^{p e r}=-\frac{2}{c^{2}} \sqrt{a \cdot G M} \cdot e \cdot \sin E,
$$

где $a$ - большая полуось эллипсоида Земли; $e$ - эксцентриситет; $E$ - эксцентрическая (угловая) аномалия орбиты спутника GPS; $c$ - скорость света; $G$ - гравитационная постоянная; $M$ - масса Земли.

Движущиеся бортовые часы спутника со временем $T_{s v}$ связаны с номинальной шкалой времени системы ГЛОНАСС преобразованием, полученным согласно общей теории относительности,

$$
\frac{d T_{s v}}{d t}=1-\frac{\left[V(x, y, z)-W_{0}+\Delta V(x, y, z)+v^{2} / c^{2}\right]}{c^{2}},
$$

где $V$ - гравитационные и приливные потенциалы Земли; $\Delta V$ - гравитационные и приливные потенциалы из-за Луны и Солнца в точке НКА с координатами $(x, y, z)$ и скоростью движения $v$; $W_{0}$ - потенциал геоида от среднего уровня моря, является константой и эквивалентен среднему радиусу Земли $R=G M / W_{0}$.

В уравнении (9) гравитационный потенциал Земли описывается как

$$
V(x, y, z)=G M / r-R,
$$

где $r$ - радиус орбиты спутника; $R$ - потенциал возмущения, определяемый как

$$
R=G M \frac{a^{2} E}{r^{3}} J_{2}\left(-\frac{3}{4} \sin ^{2} i \cos 2 u+\frac{3}{4} \sin ^{2} i-\frac{1}{2}\right),
$$

где $i$ и $u$ - отклонение орбит и аргумента широты $u=(\omega+f)$, т. е. сумма истинной аномалии $f$ и угла перигея $w$.

Квадрат скорости движения НКА можно определить отношением Кеплеровских орбит движения

$$
v^{2}=G M(2 / r-1 / a)
$$

После интегрирования (9) получаем абсолютную релятивистскую временную поправку

$$
t=T_{s v}-\Delta t^{r e l}=T_{s v}-\frac{G M}{c^{2}}\left\{\left(1 / R_{0}\right) t+\int[1 / 2 a-2 / r+R / G M-\Delta V / G M] d t\right\}
$$

На уровне пикосекунд потенциалом прилива Луны, Солнца и других планет $\Delta V(x, y, z)$ можно пренебречь.

Наиболее значительные возмущения орбит навигационных космических аппаратов ГЛОНАСС вызывается влиянием второй зональной гармоникой $J_{2}$. Следующие наиболее значительные возмущения на порядок меньше и возникают в результате притяжения Солнца и Луны. Остальные возмущения орбит спутников еще более меньшего порядка, чем от Солнца и Луны. 
Вывод. При формировании псевдодальности в имитаторе спутниковых радионавигационных сигналов ГЛОНАСС для наиболее точной проверки работоспособности навигационной аппаратуры потребителя и отладки высокоточных алгоритмов обработки радионавигационных сигналов необходимо вносить поправочный коэффициент, обусловленный релятивистским эффектом, который может достигать единиц метров. Данный поправочный коэффициент формируется для каждого навигационного космического аппарата.

\section{Список литературы / References}

[1] Sushkin I. N., Khudonogov D. Yu. Control and test bench of goniometric satellite navigation receiver. International Siberian Conference on Control and Communications (SIBCON) 2016 IEEE10.1109/SIBCON.2016.7491723

[2] Sushkin I. N., Korshunov D.E., Ruf R.A., Grigorev A. G. Method to improve the accuracy of navigation definition, JOP Conference Series: Metrological Support of Innovative Technologies. Krasnoyarsk Science and Technology City Hall of the Russian Union of Scientific and Engineering Associations. Krasnoyarsk, 2020. P. 42055.

[3] Глобальная навигационная спутниковая система ГЛОНАСС. Интерфейсный контрольный документ. Общее описание системы с кодовым разделением сигналов. АО «Российские космические системы», Москва, 2016. 133 с. [Global navigation satellite system GLONASS. Interface control document. General description of the code division signal system. JSC «Russian Space Systems», Moscow, 2016. 133 p. (in Russian)]

[4] Rutman J. Characterization of Phase and Frequency Instabilities in Precision Frequency Sources: Fifteen Years of Progress. Proceedings of the IEEE, 1978, 66,1048-1075.

[5] Фатеев В. Ф. Релятивистская метрология околоземного пространства-времени. Монография. Менделеево: ФГУП «ВНИИФТРИ», 2017. [Fateev V. F. Relativistic metrology of near-earth space-time. Mendeleevo's monograph: FSUE «VNIIFTRI», 2017 (in Russian)] 